CERN-EP/99-30

February 19, 1999

\title{
A LARGE STREAMER CHAMBER MUON TRACKING DETECTOR IN A HIGH-FLUX FIXED-TARGET APPLICATION
}

The Spin Muon Collaboration (SMC)

\begin{abstract}
Arrays of limited streamer tubes of the Iarocci type were deployed in our experiment at CERN as part of a forward muon detector system with provisions for the beam to pass through the center of each panel in the array. A total of sixteen $4 \mathrm{mx} 4 \mathrm{~m}$ panels were assembled with inductive readout strips on both sides of each panel. An active feedback system was deployed to regulate the high voltage to the streamer tubes to insure a constant efficiency for minimum ionizing particles. The arrays were operated in this environment for over five years of data taking. Streamer tube track-reconstruction efficiencies and tube replacement rates are reported.
\end{abstract}

Submitted to NIM 
D. Adams $^{18}$, B. Adeva ${ }^{20}$, E. Arik ${ }^{2}$, A. Arvidson ${ }^{23, a}$, B. Badelek ${ }^{23,25}$, M.K. Ballintijn ${ }^{15, b}$, G. Bardin ${ }^{19, \dagger}$, G. Baum ${ }^{1}$, P. Berglund ${ }^{8}$, L. Betev ${ }^{13, f f}$, I.G. Bird ${ }^{19, c}$, R. Birsa ${ }^{22}$,

P. Björkholm ${ }^{23, \mathrm{~d}}$, B.E. Bonner ${ }^{18}$, N. de Botton ${ }^{19}$, M. Boutemeur ${ }^{26, g g}$, F. Bradamante ${ }^{22}$, A. Bravar ${ }^{11}$, A. Bressan 22, f, S. Bültmann ${ }^{1, g}$, E. Burtin ${ }^{19}$, C. Cavata ${ }^{19}$, D. Crabb ${ }^{24}$,

J. Cranshaw ${ }^{18, \mathrm{~h}}$, T. Çuhadar ${ }^{2,15}$, S. Dalla Torre ${ }^{22}$, R. van Dantzig ${ }^{15}$, B. Derro ${ }^{4}$,

A. Deshpande ${ }^{26}$, S. Dhawan ${ }^{26}$, C. Dulya ${ }^{4, \mathrm{i}}$, A. Dyring ${ }^{23, j}$, S. Eichblatt ${ }^{18, \mathrm{k}}$, J.C. Faivre ${ }^{19}$,

D. Fasching ${ }^{17,1}$, F. Feinstein ${ }^{19}$, C. Fernandez ${ }^{20,9}$, S. Forthmann ${ }^{7}$, B. Frois ${ }^{19}$, A. Gallas ${ }^{20}$,

C. Garabatos ${ }^{20, \text { hh }}$, J.A. Garzon ${ }^{20,9}$, T. Gaussiran ${ }^{18}$, H. Gilly ${ }^{6}$, M. Giorgi ${ }^{22}$,

E. von Goeler ${ }^{16}$, S. Goertz ${ }^{3}$, I.A. Golutvin ${ }^{10}$, A. Gomez-Tato ${ }^{20,9}$, G. Gracia ${ }^{20, i i}$, N. de Groot ${ }^{15, m}$, M. Grosse Perdekamp ${ }^{4, n}$, E. Gülmez ${ }^{2}$, K. Haft ${ }^{13}$, D. von Harrach ${ }^{11}$,

T. Hasegawa ${ }^{14, o}$, P. Hautle ${ }^{5, p}$, N. Hayashi ${ }^{14, q}$, C.A. Heusch ${ }^{5, r}$, N. Horikawa ${ }^{14}$, V.W. Hughes ${ }^{26}$, G. Igo ${ }^{4}$, S. Ishimoto ${ }^{14, \mathrm{~s}}$, T. Iwata ${ }^{14}$, E.M. Kabuß ${ }^{11}$, T. Kageya ${ }^{14}$, A. Karev ${ }^{10}$, H.J. Kessler ${ }^{6, j j}$, T.J. Ketel ${ }^{15}$, J. Kiryluk ${ }^{25}$, Iu.Kiryushin ${ }^{10}$, A. Kishi ${ }^{14}$, Yu. Kisselev ${ }^{10}$, L. Klostermann ${ }^{15, \mathrm{t}}$, D. Krämer ${ }^{1}$, W. Kröger ${ }^{5, \mathrm{r}}$, K. Kurek ${ }^{25}$, J. Kyynäräinen ${ }^{1,8, u}$, M. Lamanna ${ }^{22, f}$, U. Landgraf ${ }^{6}$, K. Lau ${ }^{9}$, T. Layda ${ }^{5}$, J.M. Le Goff ${ }^{19}$, F. Lehar ${ }^{19}$, A. de Lesquen ${ }^{19}$, J. Lichtenstadt ${ }^{21}$, T. Lindqvist ${ }^{23}$, M. Litmaath ${ }^{15, \mathrm{k}}$, M. Lowe ${ }^{18,1}$, A. Magnon ${ }^{19}$, G.K. Mallot ${ }^{11, \mathrm{f}}$, F. Marie ${ }^{19}$, A. Martin $^{22}$, J. Martino ${ }^{19}$, T. Matsuda ${ }^{14,0}$, B. Mayes ${ }^{9}$, J.S. McCarthy ${ }^{24}$, K. Medved ${ }^{10}$, W. Meyer ${ }^{3}$, G. van Middelkoop ${ }^{15}$, D. Miller ${ }^{17}$, Y. Miyachi ${ }^{14}, \mathrm{~K}_{\text {. Mori }}{ }^{14}$, J. Moromisato $^{16}$, J. Nassalski ${ }^{25}$, L. Naumann ${ }^{5, \dagger}$, T.O. Niinikoski ${ }^{5}$, J.E.J. Oberski ${ }^{15}$, A. Ogawa ${ }^{14}$, C. Ozben $^{2}$, D.P. Parks ${ }^{9}$, H. Pereira ${ }^{19}$, A. Penzo ${ }^{22}$, F. Perrot-Kunne ${ }^{19}$, D. Peshekhonov ${ }^{10, \mathrm{~h}}$, R. Piegaia ${ }^{5,26, \mathrm{w}}$, L. Pinsky ${ }^{9}$, S. Platchkov ${ }^{19}$, M. Plo ${ }^{20}$, D. Pose $^{10}$, H. Postma ${ }^{15}$, J. Pretz ${ }^{11, v}$, T. Pussieux ${ }^{19}$, J. Pyrlik ${ }^{9}$, G. Rädel ${ }^{5}$, I. Reyhancan ${ }^{2}$, G. Reicherz ${ }^{3}$, A. Rijllart ${ }^{5}$, J.B. Roberts ${ }^{18}$, S. Rock ${ }^{5, \mathrm{x}}$, M. Rodriguez $^{23, \mathrm{w}}$, E. Rondio $^{25}$, A. Rosado ${ }^{13}$, B. Roscherr ${ }^{26}$, I. Sabo ${ }^{21}$, J. Saborido ${ }^{20}$, A. Sandacz ${ }^{25}$, D. Sanders ${ }^{9, e e}$, I. Savin ${ }^{10}$, P. Schiavon ${ }^{22}$, A. Schiller ${ }^{7}$, K.P. Schüler ${ }^{26, y}$, R. Segel ${ }^{17}$, R. Seitz ${ }^{11, \mathrm{e}}$, Y. Semertzidis ${ }^{5, \mathrm{n}}$, S. Sergeev ${ }^{10}$, F. Sever ${ }^{15, \mathrm{z}}$, P. Shanahan ${ }^{17, \mathrm{k}}$, E. P. Sichtermann ${ }^{15}$, F. Simeoni ${ }^{22}$, G.I. Smirnov ${ }^{10}$, A. Staude ${ }^{13}$, A. Steinmetz ${ }^{11, v}$, U. Stiegler ${ }^{5}$, H. Stuhrmann ${ }^{7}$, M. Szleper ${ }^{25}$, K.M. Teichert ${ }^{13}$, F. Tessarotto ${ }^{22}$, D. Thers ${ }^{19}$, W. Tlaczala ${ }^{25, \text { aa }}$, S. Trentalange ${ }^{4}$, A. Tripet ${ }^{1}$, I. Tzamouranis ${ }^{9, d d}$, G. Unel $^{2}$, M. Velasco ${ }^{17, f}$, J. Vogt $^{13}$, R. $\operatorname{Voss}^{5}$, R. Weinstein ${ }^{9}$, C. Whitten ${ }^{4}$, R. Windmolders ${ }^{12}$, R. Willumeit ${ }^{7}$, W. Wislicki ${ }^{25}$, A. Witzmann ${ }^{6, b b}$, N. Zamiatin ${ }^{10}$, A.M. Zanetti ${ }^{22}$, K. Zaremba ${ }^{25, \text { aa }}$, J. Zhao ${ }^{7, c c}$ 
1) University of Bielefeld, Physics Department, 33501 Bielefeld, Germany ${ }^{\text {aaa }}$

2) Bogaziçi University and Istanbul Technical University, Istanbul, Turkey ${ }^{\text {bbb }}$

3) University of Bochum, Physics Department, 44780 Bochum, Germanyaaa

4) University of California, Department of Physics, Los Angeles, 90024 CA, USA ${ }^{\text {ccc }}$

5) CERN, 1211 Geneva 23, Switzerland

6) University of Freiburg, Physics Department, 79104 Freiburg, Germany ${ }^{\text {aaa }}$

7) GKSS, 21494 Geesthacht, Germany aaa

8) Helsinki University of Technology, Low Temperature Laboratory and Institute of Particle Physics Technology, Espoo, Finland

9) University of Houston, Department of Physics, and Institute for Beam Particle Dynamics, Houston, 77204 TX, USA ${ }^{\text {ccc,ddd }}$

10) JINR, Dubna, RU-141980 Dubna, Russia

11) University of Mainz, Institute for Nuclear Physics, 55099 Mainz, Germany ${ }^{\text {aaa }}$

12) University of Mons, Faculty of Science, 7000 Mons, Belgium

13) University of Munich, Physics Department, 80799 Munich, Germany ${ }^{\text {aaa }}$

14) Nagoya University, CIRSE and Department of Physics, Furo-Cho, Chikusa-Ku, 464 Nagoya, Japan eee

15) NIKHEF, Delft University of Technology, FOM and Free University, 1009 AJ Amsterdam, The Netherlands ${ }^{\text {fff }}$

16) Northeastern University, Department of Physics, Boston, 02115 MA, USA ddd

17) Northwestern University, Department of Physics, Evanston, 60208 IL, USA ${ }^{\text {ccc,ddd }}$

18) Rice University, Bonner Laboratory, Houston, 77251-1892 TX, USA ${ }^{\text {ccc }}$

19) C.E.A. Saclay, DAPNIA, 91191 Gif-sur-Yvette, France ${ }^{\text {ggg }}$

20) University of Santiago, Department of Particle Physics, 15706 Santiago de Compostela, Spain ${ }^{\text {hhh }}$

21) Tel Aviv University, School of Physics, 69978 Tel Aviv, Israeliii

22) INFN Trieste and University of Trieste, Department of Physics, 34127 Trieste, Italy

23) Uppsala University, Department of Radiation Sciences, 75121 Uppsala, Sweden

${ }^{24)}$ University of Virginia, Department of Physics, Charlottesville, 22901 VA, USA ${ }^{\text {ccc }}$

25) Soltan Institute for Nuclear Studies and Warsaw University, 00681 Warsaw, Poland ${ }^{\mathrm{jjj}}$

26) Yale University, Department of Physics, New Haven, 06511 CT, USA ${ }^{\text {ccc }}$

a) Now at The Royal Library, 10241 Stockholm, Sweden

b) Now at Rational Software Benelux, 2132 WT Hoofddorp, The Netherlands

c) Now at CEBAF, Newport News, VA 23606, USA

d) Now at Ericsson Infocom AB, Karlstad, Sweden

e) Now at University of Montreal, H3C 3J7, Montreal, PQ, Canada

f) Now at CERN, 1211 Geneva 23, Switzerland

g) Now at University of Virginia, Department of Physics, Charlottesville, 22901 VA, USA

h) Now at Texas Tech Univ., Lubbock TX79409-1051, USA

i) Now at CIEMAT, Avda Complutense 22, 28040 Madrid, Spain

j) Now at Swedish Space Corporation, 17104 Solva, Sweden

k) Now at Fermi National Accelerator Laboratory, Batavia, 60510 Illinois, USA

1) Now at University of Wisconsin, USA

m) Now at Bristol Univ., Bristol, UK

n) Now at Brookhaven National Laboratory, Upton, 11973 NY, USA

o) Permanent address: Miyazaki University, Faculty of Engineering, 889-21 Miyazaki-Shi, Japan

p) Permanent address: Paul Scherrer Institut, 5232 Villigen, Switzerland

q) Permanent address: The Institute of Physical and Chemical Research (RIKEN), wako 351-01, Japan 


\section{Contents}

1 Introduction 4

2 Individual Streamer Tubes 4

3 Array Structure 5

$4 \quad$ High Voltage and Gas Supply 6

$5 \quad$ Stabilization of Streamer Chamber Gain via High Voltage

Feedback Loop 7

5.1 Chamber Response As a Function of Operating Voltage and Environment 7

5.2 Stabilization of the Average Streamer Charge 8

6 Readout and Data Acquisition Electronics 9

6.1 Overview 9

6.2 The D779 Streamer Tube Shift Register Integrated Circuit 9

6.3 The SGS IP32 Card Support Functions 10

6.4 SY480 Splitter Board 12

6.5 Streamer Tube Acquisition System (STAS) 12

$7 \quad$ Performance Summary 13

r) Permanent address: University of California, Institute of Particle Physics, Santa Cruz, 95064 CA, USA

s) Permanent address: KEK, Tsukuba-Shi, 305 Ibaraki-Ken, Japan

t) Now at Ericsson Telecommunication, 5120 AA Rijen, The Netherlands

u) Now at University of Jyväskylä, Dept. of Physics, FTN-40351, Jyväskylä, Finland

v) Now at Yale University, Department of Physics, New Haven, 06511 CT, USA

w) Permanent address: University of Buenos Aires, Physics Department, 1428 Buenos Aires, Argentina

x) Permanent address: The American University, Washington D.C. 20016, USA

y) Now at DESY, Hamburg, Germany

z) Now at ESFR, F-38043 Grenoble, France

aa) Permanent address: Warsaw University of Technology, 00-665 Warsaw, Poland

bb) Now at F.Hoffmann-La Roche Ltd., CH-4070 Basel, Switzerland

cc) Now at Oak Ridge Nat. Lab., Oak Ridge, TN37831-6393, USA

dd) Now at Enron, Inc., Houston, Texas, USA

ee) Now at University of Alabama at Huntsville, Huntsville, Alabama, USA

ff) Now with University of California, Department of Physics, Los Angeles, 90024 CA, USA, permanent address at CERN, 1211 Geneva 23, Switzerland

gg) Now at University of Munich, Physics Department, 80799 Munich, Germany

hh) Now at GSI, Darmstadt, Germany

ii) Now at NIKHEF, 1009 AJ Amsterdam, The Netherlands

jj) Now at SBC Warburg Dillon Read, CH-4002 Basel, Switzerland

aaa) Supported by the Bundesministerium für Bildung, Wissenschaft, Forschung und Technologie, Germany

bbb) Partially supported by TUBITAK and the Centre for Turkish-Balkan Physics Research and Application (Bogaziçi University)

ccc) Supported by the U.S. Department of Energy

ddd) Supported by the U.S. National Science Foundation

eee) Supported by Monbusho Grant-in-Aid for Scientific Research (International Scientific Research Program and Specially Promoted Research), Japan

fff) Supported by the National Science Foundation (NWO) of the Netherlands

ggg) Supported by the Commissariat à l'Energie Atomique, France

hhh) Supported by Comision Interministerial de Ciencia y Tecnologia, Spain

iii) Supported by the Israel Science Foundation

jjj) Supported by the Polish State Committee for Scientific Research (KBN) grant nr.2 P03B08114 and 2P03B13214

†) Deceased. 
7.1 Longevity of Streamer Chambers 13

7.2 Detector Efficiency 15

8 Conclusions $\mathbf{1 6}$

9 Acknowledgements 16 
This paper reports on our experience in the deployment of a large streamer tube array as part of our deep inelastic muon scattering experiment, NA47 at CERN, to study the spin-dependent structure function $g_{1}$ of the nucleon. The detector, known within our collaboration as ST6/7, was originally installed and first used to take data in 1992, and was thereafter in continuous use in data collection through the conclusion of the experiment's data taking in 1996. Located approximately $20 \mathrm{~m}$ downstream of the polarized scattering target, behind an iron absorber wall, this detector was centered on the beamline and served as the primary scattered muon tracker. The detector consisted of four $4 \mathrm{~m}$ by $4 \mathrm{~m}$ modules, each of which contained four planes of streamer tubes. Each separate streamer tube plane was fitted with a set of external inductive readout strips which were deployed on both the front and rear faces of each plane. Thus, the entire array comprised 16 planes of streamer tubes and a total of 32 planes of coordinates of strip readouts. The number and orientation of the planes were selected based on results of Monte Carlo calculations which simulated the inherent geometric inefficiency of streamer tubes due to their $\sim 85 \%$ active area as seen by a perpendicularly incident beam.

We believe this to have been the first deployment of streamer tubes in an open spectrometer in direct proximity to a high energy muon beamline in a fixed target experiment. We will describe here the novel features and accomplishments of this detector including $(a)$ the special properties of the tubes themselves along with a description of the structure in which they were deployed, $(b)$ the use of a direct feedback system to stabilize the performance of the detector, $(c)$ the design of the low-cost readout electronics system, and $(d)$ finally a summary of the performance of the detector as a whole including our experience with the longevity of the tubes under beam conditions.

\section{Individual Streamer Tubes}

The individual chambers were constructed at the Houston-Northeastern Streamer Chamber Assembly and Research Facility (SCARF), located at the University of Houston [1]. The chambers (SC's) are individual self-contained Polyvinyl-Chloride (PVC) packages that enclose PVC extrusions which in turn form eight individual $\sim 1 \mathrm{~cm}$ wide streamer tubes (ST's). Each tube encloses a single $50 \mu \mathrm{m}$ silver plated tungsten wire, in the manner of Iarocci et al. [2], including the use of 3 small plastic wire supports spaced approximately evenly along the wire. SCARF improved several key procedures for the production of these chambers, including $(a)$ the careful control of the technique used to coat the PVC by carbon DAG [3], (b) adoption of new burn-in procedures, $(c)$ development of new wiring and staking devices, $(d)$ a modification of the specifications for and inspection of the PVC, and $(e)$ the use of novel procedures for leak detection [4]. As a result of these improvements, the noise rate was greatly reduced and long high voltage plateaus were routinely achieved. Each $4.1 \mathrm{~m}$ long chamber of 8 ST's each, was sufficiently quiet that it could be employed to observe cosmic ray rates, running in a self triggered mode without any external coincidence mechanism.

Many streamer chamber production groups have operated since 1986 [5]. In general, typical high voltage plateaus reported by these groups have been in the range of 50$400 \mathrm{~V}$ [2]. In contrast, streamer chamber plateaus from chambers produced at SCARF typically averaged $700 \mathrm{~V}[1]$. Figure 1 is a SCARF plateau of the chamber $6.2 \mathrm{~m}$ long. What is shown is the cosmic ray rate vs. high voltage (HV) with no coincidence required, but with a dead time of 850 ns inserted after each detected pulse. It should be noted that after the installation of the detector and an evaluation of the readout electronics, 
the decision was taken to run on the knee, several hundred volts below the plateau to reduce the net charge collected on the wires in an effort to increase the longevity of the chambers. The use of the feedback setup which is described below made this running point sufficiently stable in efficiency.

\section{$3 \quad$ Array Structure}

To mount the chambers in the detector, PVC "super-sleeves" were employed, each containing four of the 8-wire chambers. The readout strips were bonded to the outer surfaces of these supersleeves rather than directly to the chambers, as shown in cross section in Figure 2. When a single 8-tube SC itself failed, it was removed from the supersleeve by simply sliding it out. A previously burned-in spare SC was inserted to replace it. Thus, a chamber replacement could typically be accomplished by as few as 2 persons during a single 15 minute access to the experiment without having to interfere with any of the other components of the detector.

Due to the necessity of allowing the beam to pass through the chamber, a central deadened region of approximately $8 \mathrm{~cm}$ radius was provided by manufacturing sets of special chambers with central semi-circular deadened regions of $8 \mathrm{~cm}$ radius. The deadening was accomplished by two separate techniques, each of which worked very well. One method consisted of simply potting the central region of the wired PVC extrusions with an epoxy fill. The other method required sleeving the wires in the central region with $\sim 0.5 \mathrm{~mm}$ copper tubes which were soldered in place to effectively increase the wire diameter and correspondingly dramatically decrease the gas amplification factor in that region. The second method was preferable only in that it provided considerably less material in the actual beam path which served to minimize the scattering of beam particles as seen from further downstream.

Figure 3 is a schematic side view of a typical module. A central hexcel ${ }^{T M}$ support plane carries the four layers of streamer chambers, with two layers on each side. Both of the streamer chamber layers on one side have horizontal wire orientation and the two on the opposite side are arranged vertically. Each set of supersleeves has the associated readout strip planes bonded to either side, and the two adjacent SC layers are offset perpendicular to the wire direction with respect to each other to reduce the likelihood that the extrusion walls do not align from one layer to the next. The two SC layers on each side of the hexcel ${ }^{T M}$ were further separated by a foam spacer. The spacer consisted of a $5 \mathrm{~mm}$ thick sheet of Sintrex ${ }^{T M}$ [6], a foamed polystyrene. The adhesive used to bond all of the components together was Araldit ${ }^{T M}$ AY103/HY951, a two component epoxy which cured overnight at temperatures between 25 and $30{ }^{\circ} \mathrm{C}$. The coordinate readout strips were fabricated from $1.7 \mathrm{~mm}$ G-10 fiberglass sheets, copper clad on both sides. The side adjacent to the tubes had the copper divided into individual strips approximately 1 $\mathrm{cm}$ wide by using a circular saw blade to just cut away the copper between the strips. The far side copper was left in one continuous sheet. The two readout coordinates associated with each SC layer had their strips running in different directions, and thus were capable of providing two dimensional track hit information from each individual SC layer. One set of the strips ran parallel to the tube directions with a strip centered directly over each individual wire. The strips on the other side of the supersleeve had a spacing of $1 \mathrm{~cm}$ and were aligned diagonally with tilt of 22.5 degrees with respect to the wire direction. In operation the strips have an image charge induced on them by the formation of streamers within the cell. In practice the streamers created by charged particle passage tend to spread along the wire direction. This has the affect of increasing the number of crossing 
strips which have significant charge pulses induced on them from a single hit. The width of the contiguous block of strips that exceed threshold due to the induced charge from a single incident particle is termed the cluster-size. The angle of 22.5 degrees was chosen as a compromise between minimization of this cluster size and the maximization of the ability to localize the track hit location using a stereo reconstruction technique. The output of the strips is carried by twisted pair ribbon cable to the data acquisition electronics cards which were mounted directly on the modules. One side of each twisted pair was soldered to the individual readout strip with the complementary wire being soldered to the corresponding copper ground plane on the other side of the G-10 sheet.

\section{$4 \quad$ High Voltage and Gas Supply}

The High Voltage (HV) and gas connections were made directly to the ends of the SC's themselves and there was no common electrical ground between the HV and the readout electronics. In practice the HV connections were made along the upper and beam-left edges of the detector while the electronics were mounted along the lower and beam-right edges. Gas connections were made initially to the HV input sides with "daisychain" tubes on alternating ends linking up to 10 tubes in a single series circuit. The output from the end of each daisy-chain was connected to the external gas return system where flow was monitored to expose gas leaks in the system.

The gas inputs were supplied through individually controlled lines to each $96 \mathrm{SC}$ set of horizontal and vertical SC's in each 4-plane module. Due to the higher currents toward the center of the detector, the central SC's were ganged in two daisy chains of 4 chambers each with two 10-chamber gangs on either side. The flow through each gang was roughly balanced to be equivalent. The input flows were regulated by CERN supplied flow controllers. The flow rates during running were set to generally average one volume exchange in each plane approximately every five to six hours under normal conditions. Of course the smaller central gangs would receive a proportionally greater number of exchanges when compared with the 10-tube gangs. The gas employed throughout the experiment was $75 \%$ Isobutane and $25 \%$ Argon by volume. It is worth noting that we were able to use lower purity ( $\sim 99 \%)$ Isobutane as a cost saving measure by venting the first $5 \%$ of each bottle and terminating use of each bottle with no less than $5 \%$ remaining. The performance of the SC's were not noticeably affected by this procedure when compared with their performance using the more costly high purity ( $\sim 99.9 \%)$ Isobutane.

One hundred and sixty independently controllable and readable HV channels were available, supplied by four CAMAC controllable CAEN Model SY127/A432 crates. In order to power the ST's, individual HV channels were split to supply several SC's each. The chambers near the beam operate at rates over $5 \mathrm{kHz}$. In that region fewer SC's are served per HV channel, whereas for the chambers farther away from the beam, up to 7 were served per HV channel. Minimum readable currents on each HV channel are 100 nA. Individual SC's, unless aged by high cumulative rates, have quiescent currents of less than $10 \mathrm{nA}$, so that even when $10 \mathrm{SC}$ 's are connected to one channel, the quiescent current cannot be measured on these HV supplies. In the presence of the muon beam, which nominally contained $4 \times 10^{7}$ during a 2.4 second beam spill, currents increase to approximately $100 \mu \mathrm{A} /$ chamber for the chambers adjacent to the beam. Several SC's away from the center the current is only 1/100 of that value. For optimal control, HV connections in one SC plane have the 2 central chambers connected to a single HV channel, followed respectively to each side by groups of 3, 6, and finally two groups of 7 chambers each. We used one 40 channel CAEN HV supply per module. Thus, this scheme leaves 
four spare HV channels per 40 channel power supply.

To improve the longevity of the SC's a feature of the high voltage supply that allowed multiple current limits to be used on each channel was employed. During the beam spill the current limits were set high enough to avoid trips under nominal beam conditions. For example, as already noted, currents as high as $100 \mu \mathrm{A}$ were experienced by individual central SC's during beam spills. Between spills, which typically had a total cycle time of 14.4 seconds, the limit was reduced back to the nominal beam off value. For channels that drew more beam off current than this value, the response of the power supply was to operate in a current limited mode, decreasing the voltage sufficiently to reduce the current to that limit (typically set at $2 \mu \mathrm{A}$ for each $\mathrm{HV}$ channel). The higher beam-on limit was restored early enough prior to the arrival of the next beam spill to allow the operating voltage on all of the SC's to recover to their nominal values. Evidence was seen of persistent discharges in isolated individual chambers initiated by exposure to the beam. These discharges were effectively quenched by the beam-off lower current limit. This technique allowed a few weak SC's to be used successfully which would otherwise have had to have been replaced. In many cases these high beam-off-current chambers seemed to "heal" themselves and get better over time. Individual SC's exposed to high radiation for several months may show quiescent currents which may be as much as a factor 10,000 higher than the normal quiescent current, yet their detector efficiencies did not seem to be impaired. The currents of these chambers were monitored closely for signs of approaching failure. Some of the spare HV channels were used to monitor individual SC's which exhibited signs of advanced aging. Also, as mentioned above, when an SC failed and was replaced by new chamber, the new SC was conditioned by slowly raising the applied HV. A spare HV channel was used for this "burn-in" and this was typically accomplished while the beam was on and the detector was in active data-taking use.

\section{Stabilization of Streamer Chamber Gain via High Voltage Feedback Loop}

\subsection{Chamber Response As a Function of Operating Voltage and Environment}

Outside of the already described deadened region through which the beam passes, the charged particle flux in ST6/7 was high enough that care had to be taken in selecting an operating voltage. The integrated flux in the beam halo in the vicinity of the ST6/7 detector was approximately $10 \%$ of the nominal central beam intensity. This implied the total nominal flux through the entire array was $\sim 10^{6} \mathrm{~s}^{-1}$. We were thus faced with conflicting considerations. As the voltage was increased, a gain in efficiency resulted. However, the gain in efficiency was paid for with higher chamber currents, which manifested themselves as increasing trip rate and probably reduced chamber lifetimes. In addition, the cluster size increased thereby worsening slightly the spatial resolution and the number of noise hits became larger. This can be seen in Figure 4 where the tube efficiency as well as the average pulse height and cluster size of the signals are shown as a function of the applied high voltage. Since we had no experience with the lifetime of a large streamer tube system in a high flux environment, we chose the operating voltage as low as possible, near the knee of the efficiency plateau. The benefits from this choice were small chamber current and cluster size. However, at the selected voltage (arrow in Figure 4), we could see efficiency fluctuations from day to night and as thunderstorms rolled in, as well as a gradual overall drop in efficiency as winter approached. Since the successful analysis of our data depends on being sensitive to asymmetries of a few per mil, such short term 
efficiency fluctuations could not be tolerated.

The efficiency is closely tied to the pulse height spectrum of the streamer signals through the threshold setting of the readout electronics. At the chosen high voltage, these spectra change dramatically with temperature and pressure. This is shown in Figure 5(a) for three different pressures and in Figure 5(b) for three different temperatures. In these measurements the HV was kept constant at a value corresponding to the knee of the efficiency plateau. The charge of the signals was measured with a $140 \mathrm{~ns}$ wide gate. This characterizes the signals strength relevant for the efficiency but is not the total streamer charge. The change of the spectra in Figure 5 due to different temperatures and pressures entail intolerable efficiency changes. To overcome this problem we chose the following operating philosophy: Rather than running the SC's at a constant high voltage, we chose to run them at a constant mean value of the pulse height spectrum, which is much more tightly correlated to the efficiency. The method by which this operating mode was implemented is described below.

\subsection{Stabilization of the Average Streamer Charge}

The system employed is composed of three elements: data gathering, data analysis and high voltage tuning. A dedicated small monitor 8-tube chamber and a similar control chamber, both of the same design as the ST6/7 SC's, were used to measure the integrated pulse height. These chambers were connected to the same gas system and kept at the same HV as the ST6/7 array. They were situated near ST6/7 in the beam halo and so they experienced essentially the same environmental conditions. The streamer pulse was picked up capacitively from the anode wire of the test chamber, and was measured by an ADC. The ADC gate and the readout trigger were provided by coincident hits in two scintillator counters, which sandwich the monitor and control chambers. During a machine spill, signals from about 200 halo muons were recorded. Between spills, events from a pulser-trigger were collected and used to determine the pedestal of the ADC. Also off-spill, signals from a constant current generator was used to monitor the ADC stability.

An IBM compatible PC computer reads the ADC via CAMAC. After 10,000 recorded pulses which took about 10 minutes, a correction to the present high voltage was determined. From these data the mean pulse height was calculated after proper pedestal subtraction, and then compared to a nominal value. If they differed by more than $0.8 \%$ a correction signal, together with the difference between the nominal and measured charge value, was sent to the control-computer of the experiment. The control-computer calculated a correction to the HV every 20 minutes. In order to avoid oscillations the applied correction was only $2 / 3$ of that which would be needed to bring the mean pulse height back to its nominal value in one cycle. At the end of each 20 minute period a new measurement of the average charge was initiated.

This automatic control system required built in safety features. The correct performance of the monitor chamber was ensured by comparing its response to that of the control chamber. Potential problems were excluded by the monitoring of the observed calibration charge. If there was not enough available beam to collect the required 10,000 triggers in the 20 minute cycle of the control-computer, no correction to the HV was applied. In all cases where a problem was detected a message was sent to the experiment operator. As a final safety precaution, upper and lower absolute HV limits were imposed.

A summary of the systems performance is shown in Figure 6, which displays the efficiency of the detector, evaluated with the track reconstruction software, together with the variations of the temperature, atmospheric pressure and the high voltage adjustments. 
These parameters are plotted versus time and cover a typical sub-period of SMC datataking. One can conclude from the plot that the feedback loop confines the mean value of the signal amplitude distribution within tight limits, which stabilized the track reconstruction efficiency of the streamer tube system to better than $1 \%$. The success of this system for the ST6/7 detector led to the implementation of a similar system for a second detector group the following year.

\section{$6 \quad$ Readout and Data Acquisition Electronics \\ 6.1 Overview}

The readout electronics were expressly designed for this application to provide the needed capabilities at a minimum cost per channel. The detector had 384 strips for each of the 32 sets of readout coordinates, which implies 12,288 total channels were needed to readout the entire detector. The total cost of all of the readout hardware remained below 20 CHF (Swiss Francs) per channel (in 1992 CHF).

The readout electronics consists of three basic components. The front end begins with 32 channel shift register cards which were designed by us in collaboration with SGS Thomson [7], who also manufactured the boards for us. These cards, which were designated by SGS Thomson as IP32 cards, employed their proprietary D-779 Integrated Circuits. The individual D-779's were mounted on separate surface-mount hybrid boards which also contained some of the supporting circuit elements. The IP32 cards were physically attached to the streamer tube readout strips with card edge connectors. Each card gathered inputs from 32 contiguous readout strips. The $10 \times 32 \mathrm{~cm}$ IP 32 cards were ganged on a common 20-conductor ribbon cable serial bus in chains of 12 cards (or 384 channels total), with each chain uniquely associated with a single set of readout strips, forming a single coordinate. These 12 card IP32 chains were connected via that bus to an intermediate module located in the experimental area near the detector. These modules were custom made to our specifications by CAEN [8], and were subsequently designated by them as their SY480 Splitter Boards. The individual SY480 modules were deployed in rack mountable crates of 8 modules each. Thus, each such SY480 crate was capable of servicing one entire module of four streamer chamber planes with its 8 sets of readout planes. The output of each SY480 crate was sent via two 40 conductor ribbon cables to a CAMAC module also designed for us by CAEN, and subsequently designated by them as their C267 Streamer Tube Acquisition System (STAS) CAMAC module. This setup is depicted schematically in Figure 7.

\subsection{The D779 Streamer Tube Shift Register Integrated Circuit}

The D779 streamer tube shift register chip is based on high speed CMOS technology and has been documented elsewhere [9]. Each D779 chip contains 4 analog signal input channels with a nominal input impedance of $100 \Omega$. The logical structure of the chip is shown in Figure 8. There are two separate grounds. One ground is the detector readout strip analog reference ground, and the other is the TTL digital ground. A major source of electronic noise is present due to TTL digital switching occurring on the same board with low level analog signals. Whenever a TTL device changes digital logic state, there tends to be a brief "rebound" ripple on the digital ground. Therefore, the only contact between these two grounds on the IP32 cards occurs within the D779 chip, where interference effects can be minimized. Any violation of this ground separation has the potential to introduce a noise level on the inputs. 
There is a single common threshold level input to each D779, which is referenced to the digital ground. The reference voltage must come from an external voltage source on the board, connected to the supply voltage. This can cause some difficulty during transient conditions, when the digital ground reference can shift, effectively shifting the absolute threshold reference with it. Steps can be taken to moderate such effects by using local onboard voltage regulation as a reference on each individual board.

When an input signal exceeds the threshold level on any of the 4 input channels, an internal storage digital one-shot is placed in the high state. An external clock pulse is required to regulate the digital one-shot. This clock signal is generated on each IP32 board individually by a crystal controlled oscillator. The version of the IP32 boards used in the ST6/7 array employs a $6 \mathrm{MHz}$ crystal, but the D779 chip, and the card design as a whole are rated for clock rates of up to $10 \mathrm{MHz}$. The one-shot resets on the sixth clock rise after it was originally set. Since the arrival of input hits are asynchronous with this clock, the width of the one-shot varies from a minimum of 5 clock pulses to a maximum of 6 clock pulses. This process is "non-retriggering," which implies that hits received while the one-shot is high do not reset the clocking. This in principal provides a rate limitation for the readout electronics as well as a potential for admitting background hits, as is discussed further below. A TTL pulse must arrive at the Shift Register Load (SRLD) input to the D779 while the digital one-shot is still high in order to cause the transfer of the hit information to the output shift register for that channel. In particular, it is the fall of the SRLD pulse that triggers the transfer.

The 4 one-shots on the SGS card are internally OR'ed. This OR'ed TTL level is visible at one of the outputs of the D779. The outputs of all D779's on each IP32 card are effectively OR'ed, and a single $-0.5 \mathrm{~V}$ NIM level output is provided. One may employ this digital OR in the trigger logic if necessary, although this was not done in the present application.

\subsection{The SGS IP32 Card Support Functions}

There are 4 potentiometers on each IP32 card which allow adjustment of the thresholds for the D779 chips in groups of two (i.e. in four gangs or 8 contiguous channels). The reference voltage may either be a local on-board value as mentioned above, or a global value supplied from the chain bus connecting the IP32 cards. The D779 chip requires a normal +5VDC supply (VCC), and each IP32 card has its own on board (LM7805) 5VDC regulator. The board is typically supplied with +9.5VDC power from the SY480 modules along the chain bus. In principle, +6VDC would be sufficient, but the higher supply voltage is used to insure that an overvoltage is always available to the regulators, even under the most severe fluctuations in the power bus current. Each IP32 card typically draws less than $100 \mathrm{~mA}$ under normal operations.

It is possible for a background hit to occur after the triggering event and prior to the arrival of the SRLD signal When this happens, the shift register for that background is loaded and the background hit is recorded. Since background hits increase the amount of computer time necessary to reconstruct the tracks, these are more problematic than the early hits which simply cause inefficiencies because of the non-retriggering nature of the D779's one-shots. To counter this late occurring hit background problem, a pair of external ganged one-shots are provided for each individual D779. The first of these oneshots is set with the rise of the digital OR from the D779. The second is set upon the fall of the first, and must be high for the SRLD signal to be passed to the D779. The widths of these two one-shots can be adjusted to create a delayed time window during which the 
SRLD must be received in order to reach the D779 and cause the shift registers to be loaded. This timing is shown in Figure 9. The time window is set to correspond to the trigger delay and thus for each D779 individually will act to suppress any late arriving background hits. The creation of a dead-time for each D779 individually by early arriving background hits is not affected, and still represents a limitation to the maximum rate achievable. With a $10 \mathrm{MHz}$ clock driving the one-shots, 600ns are required as a minimum between distinguishable hits on the same D779. With the $6 \mathrm{MHz}$ clock used during the SMC experiment this value was $1 \mu$ sec.

Once the SRLD has been received, the shift registers in each D779 are loaded, and are ready to be readout. This is accomplished by applying a shift clock pulse to the shift register (SR) clock input of the D779's. The shift register data is shifted out via the SROUT output of the D779, and the SRIN inputs data from farther up the shift register chain. All of the D779s on each IP32 card are daisy-chained into an effective 32-bit shift register when viewed by the IP32 input and output. The data state is determined on the rising edge of each SR clock pulse. The SR clock speed can be up to $4 \mathrm{MHz}$.

The D779's also require an occasional REFRESH pulse as an input to adjust the offset null of the sense amplifiers. This pulse must be a minimum of 500-800 ns wide and for the SGS-Thomson IP32 card, it is nominally $1 \mathrm{~s}$ wide at $30-40 \mathrm{~Hz}$. During the REFRESH pulse, the inputs are disabled producing an effective $3 \times 10^{-5}$ dead time.

The IP32's circuitry also provides an input amplifier stage as a preamp for the D779's input. Figure 10 shows the schematic for the typical preamp. R1 and C1 provide a lowpass filter with a time constant of about $30 \mathrm{~ns}$. This acts as an effective charge integrator for the input pulse. The streamer tube readout strips are electrically isolated and neutral, but as a streamer (negative charge) forms in the tubes, the image charge induced on the readout strip will be a local positive pulse. If one integrated the entire input activity from a single strip, it would clearly be charge neutral. However, the extreme asymmetry in the pulse shape allows one to effectively treat it as a $50 \mathrm{~ns}$ positive pulse. In reality, the end of the readout strip opposite to the card is open and unterminated, leading to non-inverting reflections. The width of diagonal strips is different from that of the parallel strips, and the lengths of the diagonal strips vary considerably. The cables connecting the strips to the input card edge connectors of the IP32s are $100 \Omega$ twisted pair to impedance match the hybrid inputs, but cannot universally match the transmission line impedance's of the strips. Further, the mechanical construction did not achieve a uniformity in distance (or glue thickness) over the length of the strips to better than perhaps as much as $0.1-0.5$ $\mathrm{mm}$. All of these effects cause distortions to the observed waveforms arriving at the input to the preamps. However, the underlying charge asymmetric pulse shape can be recovered with this charge integrating front end. The nominal amplification of the input pulse as seen at the D779 inputs is 8.38. Thus the effect of the threshold as applied to the hybrid (threshold) input is to divide that by 8.38 . So, for example a $25 \mathrm{mV}$ Threshold on the IP32 would admit approximately $3 \mathrm{mV}$ signals to the input of the D779.

As noted elsewhere in this paper, the decision to run the streamer tubes below their nominal plateau high voltage was made after the electronics had been constructed and installed. On plateau one can typically set the actual threshold at the D779 to $5 \mathrm{mV}$, which was the original baseline design specification for the IP32 boards. However, we typically ran them with the thresholds below $2 \mathrm{mV}$ and we did not encounter any loss of efficiency supporting the streamer tube operation with the high voltages set below their nominal plateau values.

The IP32 cards also provided injection points for placing pulses at the inputs on 
all even channels, and another injection point for all odd channels. Finally, the jumpers on the cards allowed the vectoring of the shift register inputs and outputs sufficient to enable the shift to be made along the chain card by card in either direction, although within each card the shift direction was fixed.

\subsection{SY480 Splitter Board}

As already noted, the SY480 splitter modules provided regulated power to IP32 cards via the chain bus. An external threshold reference voltage is also supplied and may be used to adjust the entire chains input thresholds providing all of the individual IP32 cards in the chain have their jumpers set to enable the external threshold reference.

A total of 5 signals are passed between the STAS module in the remote CAMAC crate and the IP32 chain by the SY480 modules. Three of the signals: the Shift Register Clock pulses (SRCK), the Shift Register Load (SRLD), and the Test Pattern (TPATT), pass from the STAS through the SY480 and on to the chain. The other two signals: the data (DATA) pulses from the shift registers, and a Data Acknowledge (DTACK) which is a mirror of the SRCK signal in synch with the actual DATA pulses, are returned from the chains through the SY480 to the STAS. All five of these signals are processed through HP2630 opto-couplers as shown in Figure 11 to isolate the circuits and the grounds in the experimental hall from those in the data acquisition barrack.

\subsection{Streamer Tube Acquisition System (STAS)}

Each STAS is capable of supporting eight separate chains. All of the STAS's functions and controls may be accessed and set via CAMAC. In normal operation, the experiment trigger is applied to the input of the STAS from which it is distributed as the SRLD signal, after about a 100 ns delay, to each of the eight chains that have been enabled via CAMAC to be in operation. The individual chains can be enabled and disabled separately. Then after a few $\mu \mathrm{s}$, a predetermined number of clock pulses are applied to the SRCK lines of the enabled chains. The number of clock pulses is based upon the length of the chain which is another of the CAMAC settable parameters within the STAS. The SRCK may be set by jumpers within the STAS to frequencies of $0.5,1,2$, and $4 \mathrm{MHz}$. We encountered no problems operating at $4 \mathrm{MHz}$. The TPATT line is modulated in synchronization with the SRCK through a cycling 8-bit pattern which also is an independent CAMAC settable parameter for each chain. The TPATT is vectored by the jumpers on the IP32 cards to act as the input to the most remote card in the chain. The number of channels read back by the STAS is actually 32 more than the number in the chain, the final 32 then being 4 repetitions of the TPATT sequence, verifying that the chain shift process is functioning properly.

Upon return the DATA lines are read in synchronization with the pulses on the corresponding DTACK lines, and bear no time synchronization with the original SRCK signal. Thus there is no timing constraint upon the length of the lines from the STAS to the chains. Note that the arrival delay of the SRLD signal can become problematic if it has to go from the experiment trigger and up to the STAS, then out to the detector. This can be overcome by providing a fast trigger directly to the SY480 modules which have been modified by us to accept such a signal and then OR it with each arriving SRLD from the STAS. This fast trigger then has to remain high until the SRLD arrives from the STAS to continue the cycle under its control. Note the drop of the SRLD initiates a fast clear and resets the IP32 cards to resume taking data. 
As each DATA shift is read, an automatic sparcification occurs. When a hit is shifted in, a counter is set, and up to 7 successive hits may be counted. When the first non-hit shift occurs after a string of hits, the prior information is formed into a single 16-bit word which encodes the address of the last hit and the width of the contiguous cluster of hits. The chain number is also encoded in each such data word. Clusters of greater than 7 contiguous hits require more than one word. These data is stored in memory buffers $4096 \times 16$-bit words deep for each channel. A separator word is placed between successive events. In practice during the SMC, each STAS was always readout after each trigger. The data words sent via CAMAC include a separator word identifying each chain followed by the data from that chain. Empty chains have a single dummy null data word. The entire system was quite robust and was operated for much of the time with only "novice" experts on duty. We managed to keep the system operational virtually continuously with only about 24 spare IP32 cards to support the 384 deployed cards and a single spare STAS to support the 4 normally in use. We had only 5 spare SY480 modules to support 32 in use. The only tense moments occurred on two occasions when we had to send one of the STAS modules to CAEN to be repaired. At no time did the SMC experience any significant loss of running time due to ST67 electronics problems. At all other times we had plenty of spare components available to maintain the system in full working order. Overall, it was reliable and troublefree.

\section{$7 \quad$ Performance Summary}

\subsection{Longevity of Streamer Chambers}

As noted initially, the SMC experiment ran at CERN over 5 periods of about 120 days per year from 1992 through 1996 [10], and during that entire time the ST67 detector was essentially in continuous service. From that experience it is possible to assess the longevity of the individual streamer chambers under this type of secondary-beamline, fixed-target running condition.

Figure 12 shows the number of individual SC's that were replaced, both as a function of year and as a function of location in the array with respect to the beam. The first comment that is in order concerns the very large number of chambers replaced during the initial period of operation (105 chambers in 1991-92) as seen in Figure 12(b). This large effect could possibly have been due to an "infant mortality" phenomenon. A certain fraction of these failures might have been caused by defects that were the result of latent fabrication flaws or by damage sustained in shipping and handling. Undoubtedly some of these initial losses were of that nature. However, given the testing procedures used at SCARF and our experience with subsequent installation of replacement chambers, most of which came from the same general production lots and were shipped and handled alongside of the initially deployed chambers, such losses were too infrequent to explain the high number of initial replacements.

Rather, we feel that a large measure of this aberration was due to significant changes in our behavior as we became more experienced with the use of the chambers in this type of high flux environment. For example, initially we were reluctant to raise the beam-off HV trip current levels for SC's which developed higher dark currents. We typically removed such chambers and discarded them, inserting a new SC in their place. With experience we learned that very often such behavior was transient, and that continuing to run the chambers with elevated current trip levels served to allow them to "heal" themselves. We also learned that there was no noticeable loss of data efficiency by following such a procedure. In addition, our handling of the operation of the chambers became more 
sensitive to hazardous situations. Thus the performance in 1993, 1994, and 1995 reflects such a strategy. Again in 1996, with the end of SMC running being anticipated, we replaced more SC's in preparation for the running period, and some fraction of the increased replacements in 1996 is due to that initial precaution. Nevertheless, there is a clear trend towards a slight annual increase in the number of chambers being replaced through the end of SMC data taking in 1996.

A second observation that can be made regarding chamber losses concerns the distribution of losses in proximity to the beam. Figure 12(a) clearly shows a tendency to lose chambers within $16 \mathrm{~cm}$ of the beam centerline. Beyond that distance however, the distribution is essentially flat and roughly comparable in total integrated flux to the total beam flux. It is clear that the total flux passing through the chambers that were closest to the beam was substantially greater than that passing through the chambers beyond that region. We estimate that the flux in the beam halo was as high as $10,000 \mathrm{~cm}^{-2} \mathrm{~s}^{-1}$ in the innermost SC's at the edge of the deadened regions which extrapolates over the full period to a flux of $\sim 5 \times 10^{10} \mathrm{~cm}^{-2}$ at $8 \mathrm{~cm}$ from the beam axis. A reasonable approximation of the halo flux at a distance $\mathrm{r}$ (in $\mathrm{cm}$ ) from the beam is given by $2 \times 10^{6} / \mathrm{r}^{5 / 2} \mathrm{~cm}^{-2} \mathrm{~s}^{-1}$. The integrated fluxes can be obtained from this formula by multiplying by our effective total exposure time of $4.8 \times 10^{6} \mathrm{~s}$ of beam.

We can also estimate the the more relevant parameter of integrated charge on the individual streamer tube wires by using the average conditions reflected by currents drawn by the chambers from their HV supplies. In effect, it amounts to using the streamers themselves as crude ion chambers. By comparing the currents drawn by the chambers in the low flux halo regions up to $2 \mathrm{~m}$ from the beam with those progressively closer to the beam we can estimate 2 parameters. First, by integrating the total running time multiplied by the nominal weighted sums of the beam-on and beam-off currents, and dividing by the number of chambers used in that location during the course of the entire experiment, we can obtain a crude estimate of the total average charge per chamber at failure. Since most failures tend to be due to localized damage within the chamber, it is perhaps more appropriate to estimate the local integrated average charge density per $\mathrm{cm}^{2}$ at the point of closest approach to the beam of the active region of each chamber. Rather than attempt to analyze the distribution of hits from random triggers, we can also analyze the distribution of HV supply currents, making the rough approximation that when integrated they are proportional to the integrated charge. This is more likely to be true at the lower flux levels farther from the beam, but at the higher flux levels closer to the beam there may be saturation effects which artificially suppress the count [11]. Thus, the relative flux estimates based upon this type of procedure will be conservative for the more central chambers.

Such an analysis suggests that the central chambers were failing at total integrated charge flows of 180-200 Coulomb per chamber in the central-most regions. Recall that each chamber contains 8 individual streamer tubes. In these chambers the maximum integrated charge collection per $\mathrm{cm}^{2}$ is estimated to have reached between 5 and 10 Coulomb $/ \mathrm{cm}^{2}$ at the time of failure. These exposure levels however do not represent the absolute failure point, but rather roughly a $50 \%$ probability of failure because of the number of tubes that have survived at these levels. In the regions farther from the beam, the failures occurred at exposure levels closer to a few Coulombs per $\mathrm{cm}^{2}$, but with a far smaller probability perhaps only in the range of $10 \%$.

As a final remark, the simplest arithmetic shows that with a total of 768 chambers deployed in the array initially, a grand total of about 150 chambers or just under $20 \%$ 
were replaced between 1991 and 1996. If one ignores the 1991-92 replacements, the average number of replacements totaled less that $1.5 \%$ of the deployed total per year.

\subsection{Detector Efficiency}

A detailed discussion of the track reconstruction algorithms employed during the SMC experiment is beyond the scope of this paper. We present here only a brief overview of the technique employed and our experience with the efficiencies of the individual chamber layers [12].

Due to the existence of the the walls between the streamer tubes and the walls of the chamber and supersleeve enclosures, only about $85.9 \%$ of the frontal area of the detector is sensitive volume for tracks with normal incidence.[13] The measured variation in this value had a standard deviation of only $0.83 \%$ due to construction differences from plane to plane. Although the overlaps of the dead regions in adjacent planes were designed to be minimized, in practice about $5 \%$ of the adjacent net dead areas did overlap (i.e. $5 \%$ of the $14.1 \%$ geometrically dead area in a typical plane was also masked by a similar adjacent dead area in the immediately adjacent similar plane). The measured efficiency is also influenced by slight inefficiencies in the vicinity of the internal chamber plastic wire supports.

There are two general contributions to the net efficiency to get a hit when a particle traverses a plane. First there is the probability that a streamer will be formed, and second, if a streamer forms, there is a subsequent probability that the signal induced on the readout strip will cause a bit to be loaded into the shift register. We have determined that the first probability is on the order of $90 \%$ for tracks that enter the sensitive volume. In practice the product of the geometric inefficiency and the streamer production probability are combined. That product typically gave a combined efficiency to form a streamer at or above $80 \%$ for fluxes of up to about $2000 \mathrm{~cm}^{-2} \mathrm{~s}^{-1}$. This value dropped roughly linearly to about $74 \%$ at a flux of $6000 \mathrm{~cm}^{-2} \mathrm{~s}^{-1}$, which occurred at about $2 \mathrm{~cm}$ from the edge of the deadened regions or about $10 \mathrm{~cm}$ from the beam. This is the highest flux value for which we have reliable efficiency data. The corresponding readout efficiencies are between 90 and $95 \%$ at the lowest fluxes, where the variations are due to many factors. There was about a $3 \%$ systematically lower readout efficiency for the diagonal coordinates with respect the corresponding parallel strips on the opposite side of the same ST plane. At the highest fluxes the readout efficiency dropped down to about $84 \%$. A potential explanation for this reduction is the decrease in effective voltage by the larger net currents drawn during the spill at the higher fluxes. Combining these effects, one obtains a net efficiency to produce a hit in a given coordinate of close to $80 \%$ at fluxes below $2000 \mathrm{~cm}^{-2} \mathrm{~s}^{-1}$, and of about $65 \%$ at a flux of $6000 \mathrm{~cm}^{-2} \mathrm{~s}^{-1}$. Figure 13 shows an efficiency plot for a single plane of SC's as a function of the track's crossing point in the plane of the wires. The view is transverse to the wire direction. The crossing points of the incident muon tracks were determined for this plot from selected events using the other 31 coordinates. The value plotted is the combined geometric and streamer formation efficiency.

As noted elsewhere in this paper, typically the streamers produced hits in several adjacent strips in each coordinate. In about $90 \%$ of the hits the cluster size was either only one or two strips, with the distribution being slightly larger for the diagonal strips. Given the organization of the detector into 4 geometrically distinct modules, each containing 4 separate independent coordinates with two sets of strips for each coordinate, we chose to use a track reconstruction technique to take advantage of that arrangement. Using information available from the external scintillator hodoscopes, we isolated regions of interest 
that were aligned along the projected paths indicated by the trigger data. Within those regions in each module, multiple coordinate intersections which we refer to as "multipoints" were searched for. The minimum requirement for a multipoint was the proximate (within $\sim 1 \mathrm{~cm}$ ) crossing of hits from a minimum of 4 coordinates, at least 3 of which were non-parallel. Once multipoints had been determined in each module, potential tracks were identified by minimum allignment requirements for at least three multipoints. Once such candidates were identified, more conventional chi-squared minimization techniques were used to fit the track to the individual coordinates included within the multipoints. There were also arbitrary criteria used to abort the analysis of individual events in the interest of saving computer time. Any event that contained more than 20 multipoints in one coordinate or more than 10 candidate tracks was aborted and not analysed further. This procedure was carefully checked to insure that it did not introduce any false asymmetries into the SMC data.

Overall in the SMC experiment we were able to reconstruct muon tracks for $\sim 90 \%$ of all raw primary muon triggers. A careful analysis of the excluded events indicates that for all events in which a clean muon track was present, the net track reconstruction efficiency was on the order of $\sim 99 \%$. In addition, we achieved a spatial resolution of $\sim 2.5$ $\mathrm{mm}$ at the front face of the detector array, and an angular resolution of $\sim 0.5 \mathrm{mrad}$ for tracks passing through the entire array under nominal running conditions.

\section{Conclusions}

We have deployed a large area streamer chamber array in a high flux fixed target experiment in general proximity to the beam, and successfully operated it throughout the entire running of the SMC experiment. We have also succeeded in stabilizing the efficiency of the detector to an absolute range of $\sim 1 \%$. The streamer chamber arrays and the associated readout system we developed turned out to be one of the most robust and reliable detector systems of the SMC experiment. We wish to acknowledge the assistance in the design and development of the electronics of both SGS Thomson and CAEN.

\section{$9 \quad$ Acknowledgements}

We wish to acknowledge the assistance in the design and development of the electronics of S. Elia, M. Grassi, S. Pedretti, W. Sangalli, and G. Scrocci from SGS Thomson's Subsystems, Engineering \& R.F. Division in (Colleoni) Agrate, Italy; C. Planzo of SGS Thomson's Geneva office; as well as G. Franchi, M. Givoletti, and F. Vivaldi of CAEN in Viareggio, Italy. We also gratefully acknowledge the loan of some readout boards during our early deployment from S. Cavestro of the University of Paduova and from L. Votano of the Laboratori Nazionali di Frascati. We acknowledge the help and assistance of all of the CERN support staff, too numerous to list, who have eased our burdens and provided essential assistance. Among the personnel at CERN, a special thank you must go to Y. Camp, E. (Liz) Cumiskey (now deceased), J-M Demolis, and L. Veronneau. Specific funding support for the ST67 detector hardware was provided by the U.S. Department of Energy, the U.S. National Science Foundation, and the Bundesministerium für Bildung, Wissenschaft, Forschung und Technologie. 


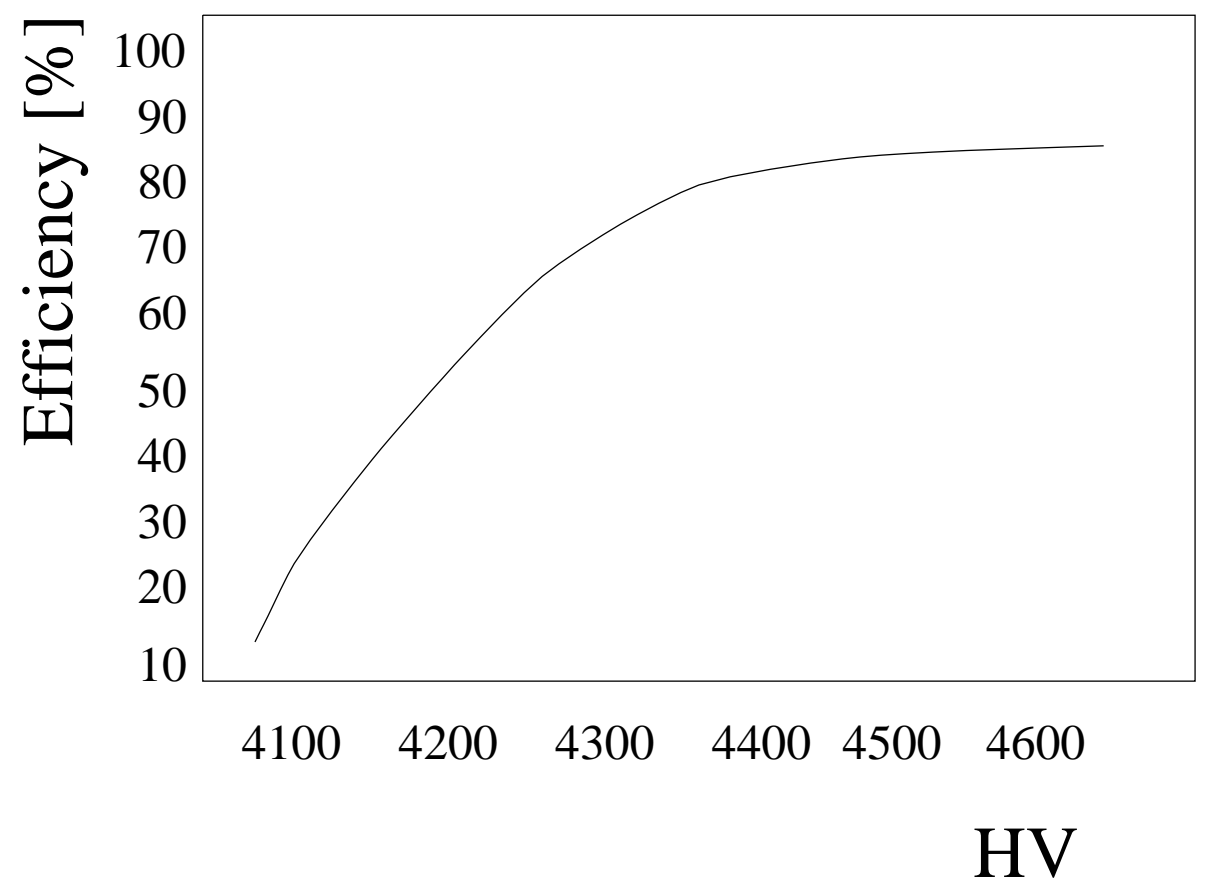

Figure 1: A typical SCARF high voltage plateau of 700 Volt from cosmic rays for a $6.2 \mathrm{~m}$ long chamber.

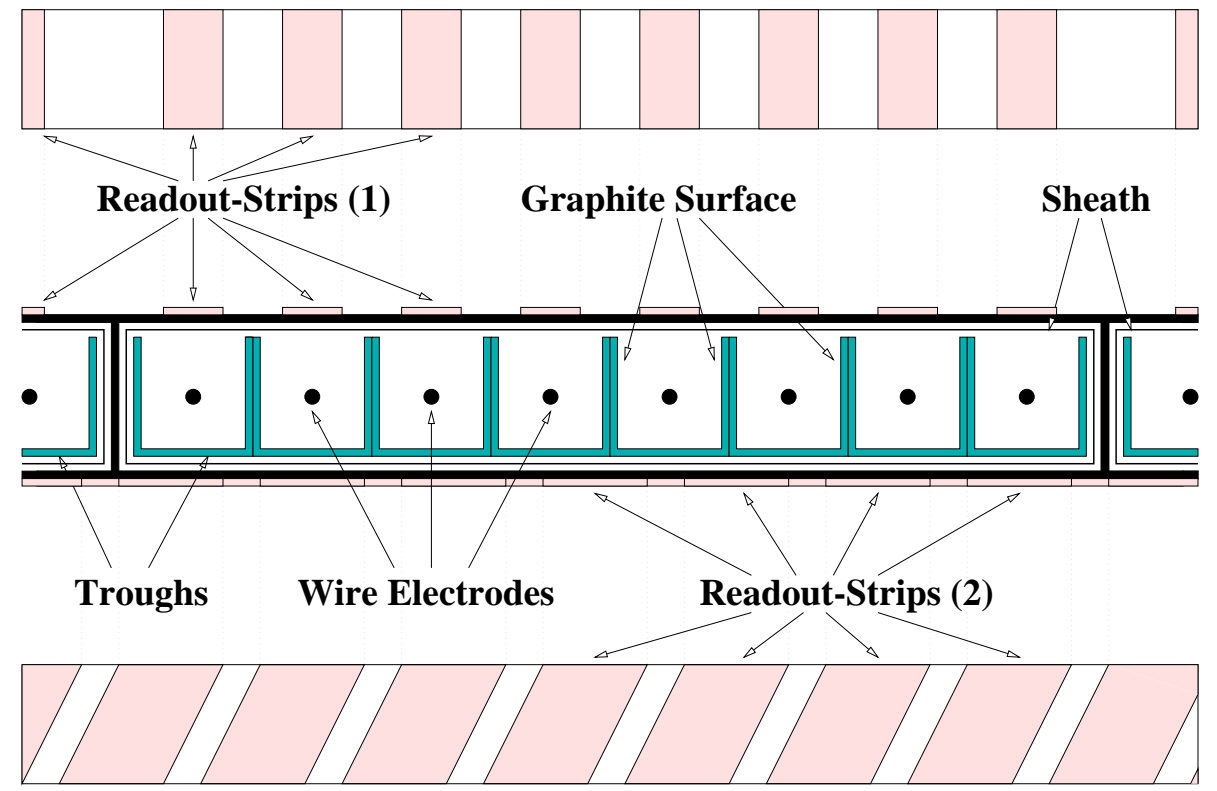

Figure 2: The cross section of a chamber consisting of 8 streamer tubes within the supersleeve sheath. The readout strips were constructed from thin double sided copper-clad G-10 sheets with the strip pattern cut into one side. The strip side of these G-10 sheets was bonded directly to the exterior of the supersleeves, which allowed removal of the individual chambers contained inside without any effect upon the strip structure. The read-out strips (1) on one side ran parallel to the wires and on the other side (2) they were slanted at an angle of $22.5^{\circ}$. 


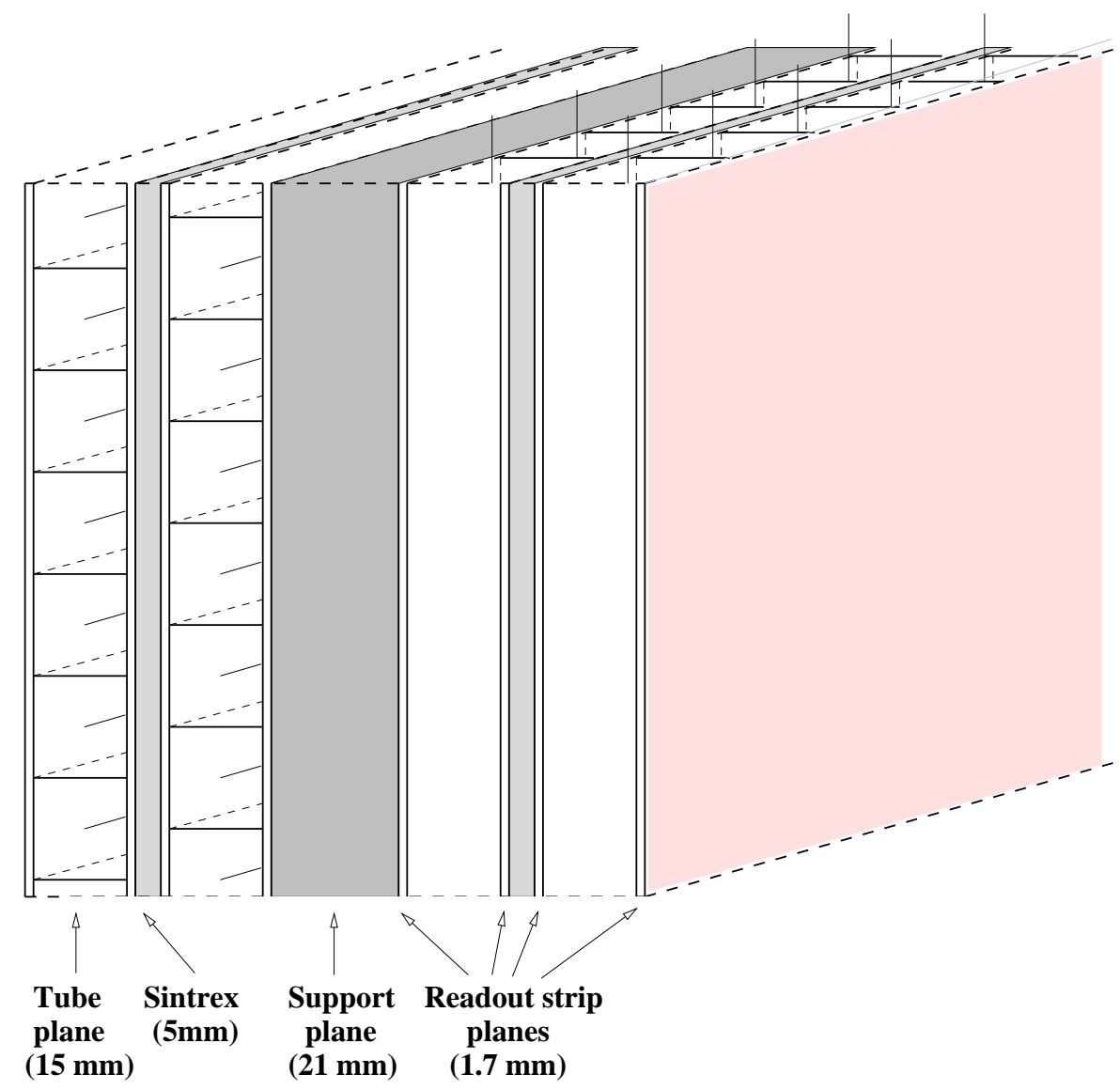

Figure 3: A schematic side view of a typical module containing 4 layers of streamer chambers and 8 planes of readout strips. There were four such modules employed during the SMC experiment. 




Figure 4: The detector efficiency $\epsilon$ is plotted vs. the voltage of the anode wires of the streamer tubes (full circles). Also shown are the average pulse charge $\langle Q\rangle$ in relative number of $A D C$ channels (open squares), and the average cluster size in number of strips (open circles). The arrow indicates the chosen operating voltage. 


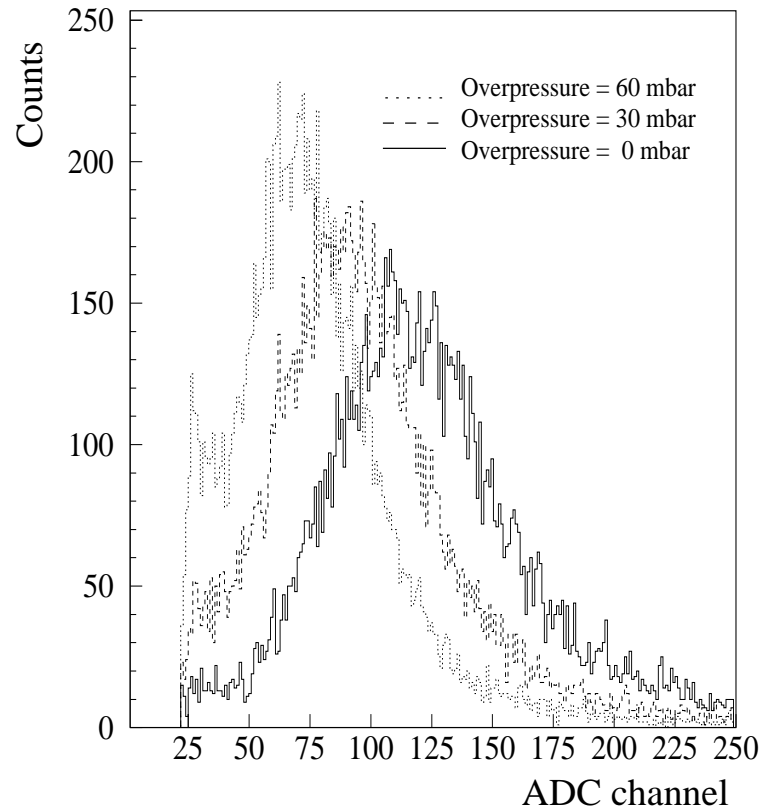

(a)

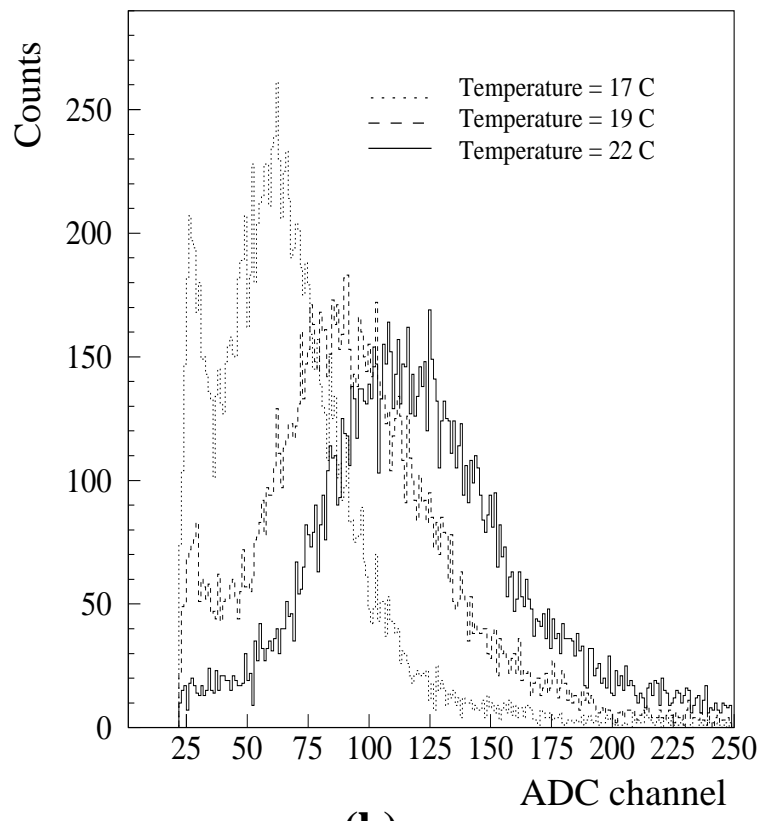

(b)

Figure 5: (a) The pulse height spectrum for muons is shown for 760 mbar and for overpressures above 760 mbar of 30 mbar and 60 mbar. All measurements were taken at the same $18^{\circ} \mathrm{C}$ ambient temperature. (b) The pulse height spectrum is shown for temperatures $17^{\circ} \mathrm{C}, 19.0^{\circ} \mathrm{C}$, and 22. $0^{\circ} \mathrm{C}$. All measurements were taken at a pressure of 760 mbar and with a $\mathrm{HV}$ setting of $4.60 \mathrm{kV}$. 


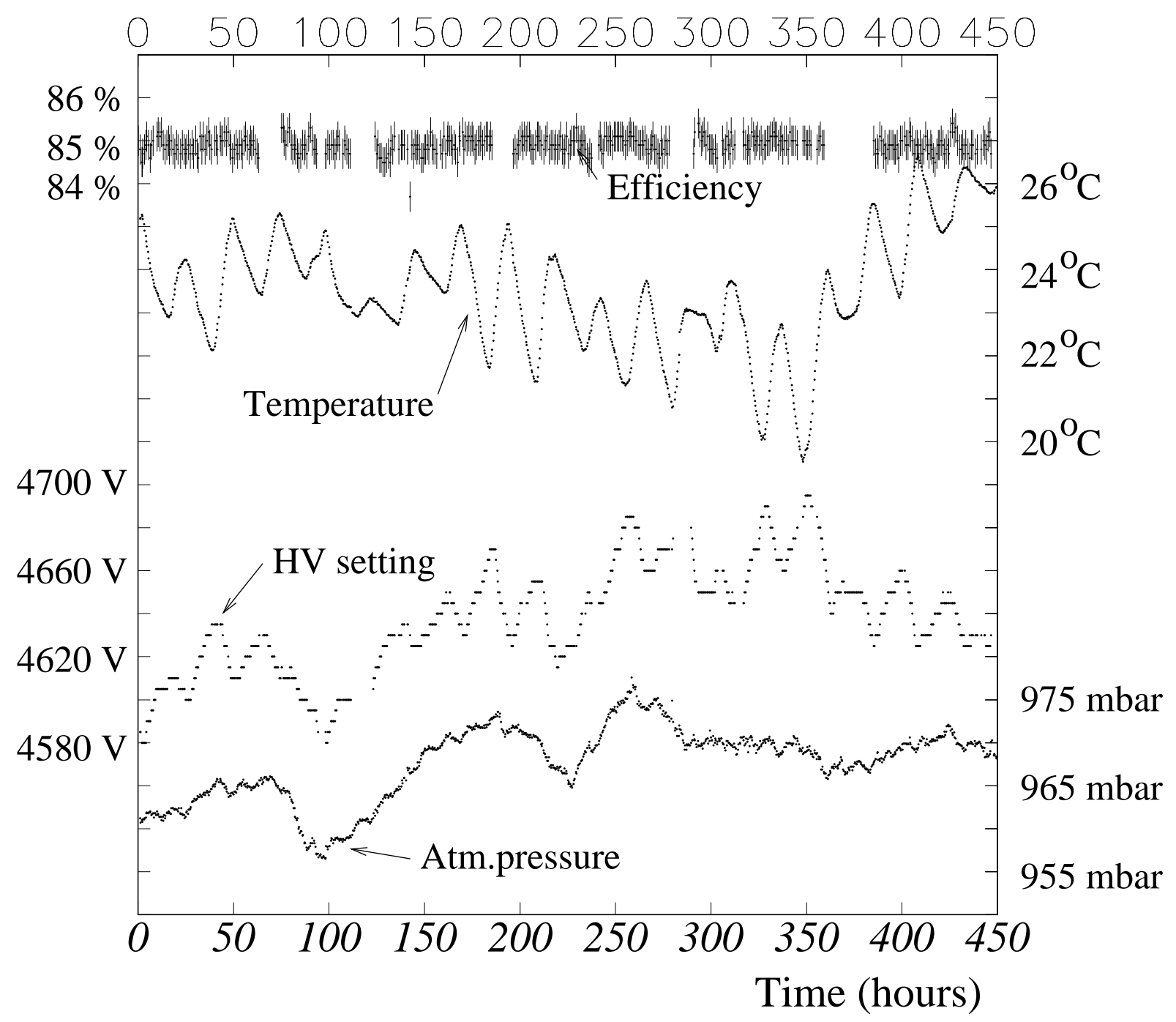

Figure 6: The efficiency of the detector based upon the subsequent off-line SMC track reconstruction software is plotted over a typical 450 hour data-taking period. Also plotted for reference are the measured ambient temperature and pressure, as well as the HV setting chosen by the feedback hardware. 


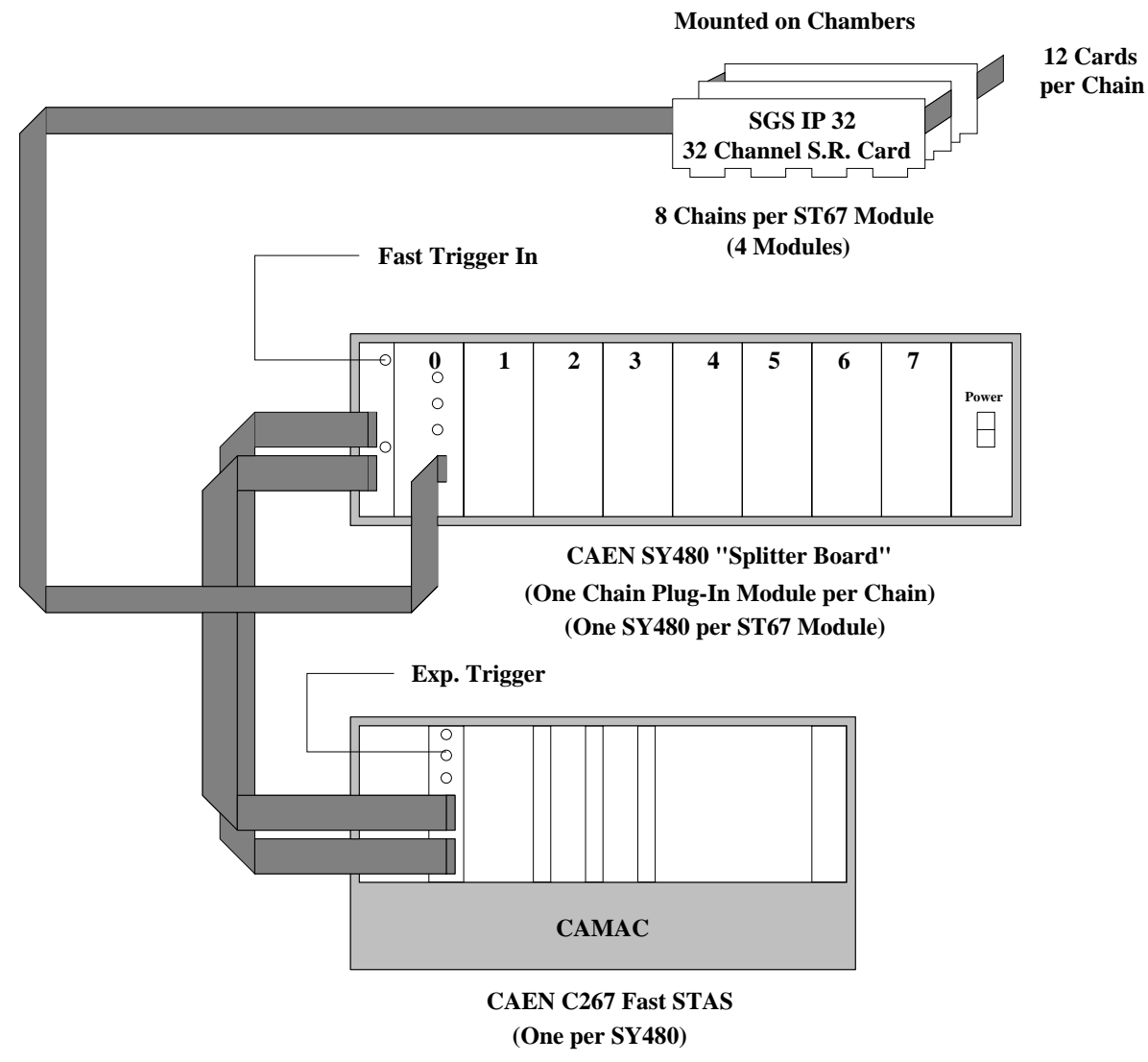

Figure 7: A block diagram of the ST67 readout electronics showing the three main components, the SGS IP32 cards which were mounted on the detector, the CAEN SY480 which was located in the hall next to the detector, and the CAEN C267 STAS which was located in the electronics barrack $\sim 30$ cable meters away.

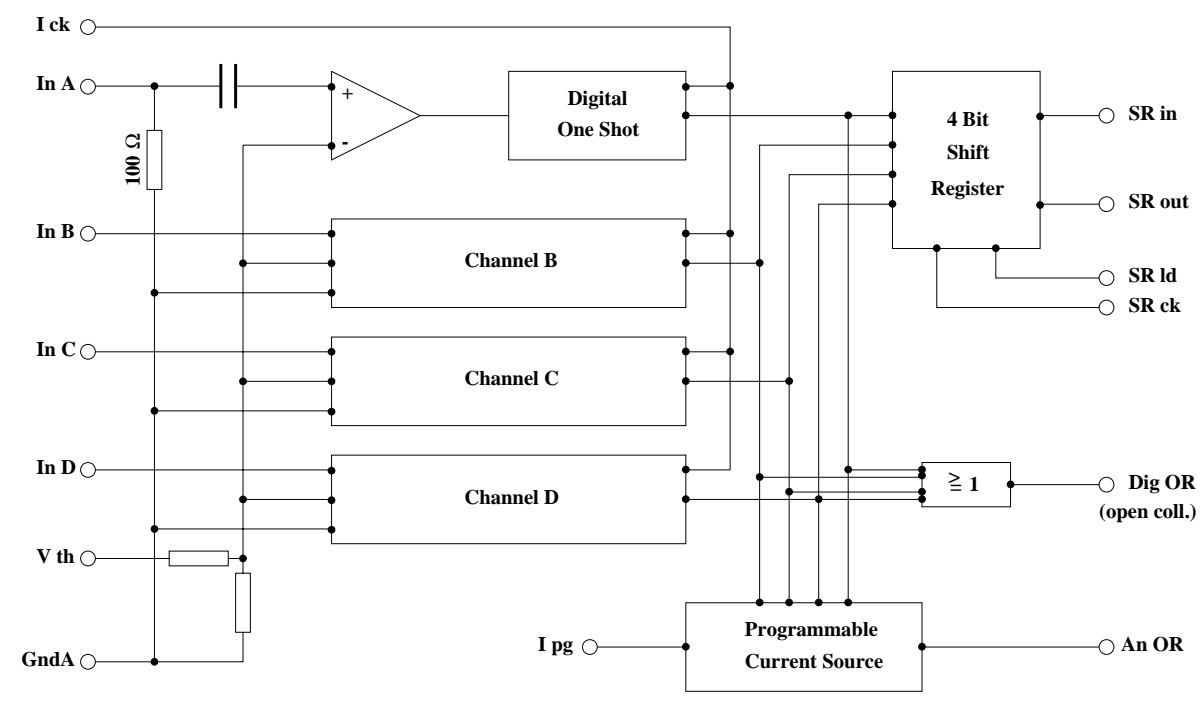

Figure 8: The logical structure of the D779 four-channel IC is shown. These were deployed on small surface mount boards that also contained the local trigger coincidence logic and the 4 individual input preamps. 




Figure 9: The relative timing of the Dr79's DATA digital one-shot is shown with respect to the IP32's DELAY and SHOT timings. The DELAY width is adjusted to set the SHOT window at the proper time to be coincident with the arrival of the LOAD signal from the experiment trigger which must fall while the DATA is still high to effect a transfer to the readout shift registers. The Shot width is adjusted to account for the maximum variation in the LOAD's arrival with respect to the DATA's rise due to among other factors the different transit times for signals at different strip distances from the readout end. This arrangement allows one to choose between the extremes of too many out-of-time background hits and an increased deadtime for individual groups of four channels.

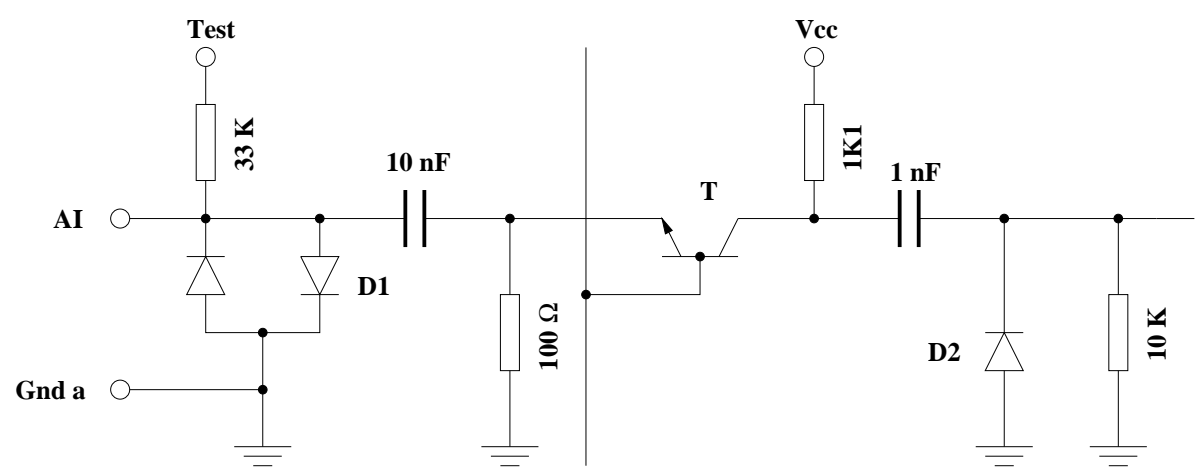

Figure 10: This preamp circuit was employed on each individual input. Note the provision for injection of test pulses using the $S Y 480$ to distribute them. The provision was made for injecting pulses into all even number channels or alternatively into all odd numbered channels to allow testing for crosstalk between adjacent channels, as well as to test the response of each channel individually. 

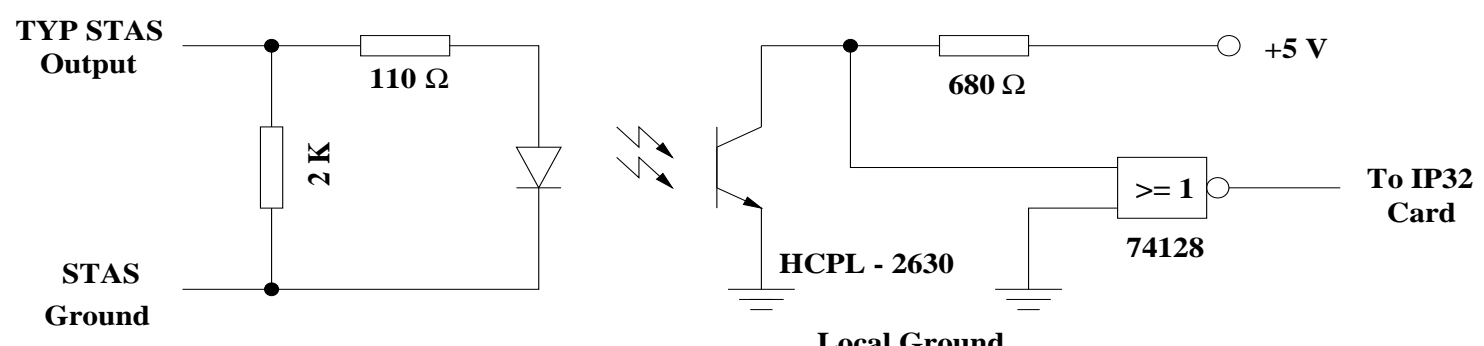

(a)

Local Ground
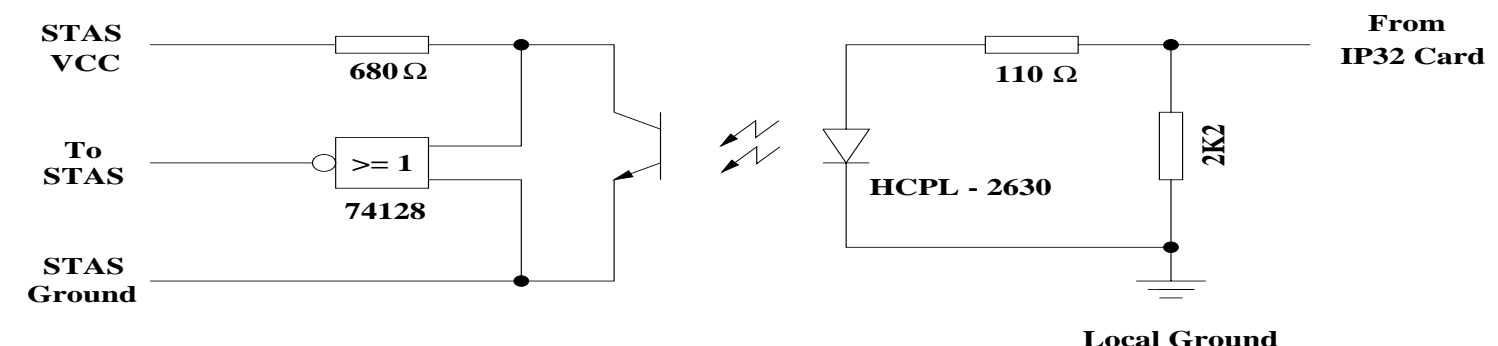

(b)

Figure 11: The HCPL 2630 Opto-couplers were deployed for all signals passing between the STAS and the SY480 in order to allow absolute electrical isolation between the electronics on or near the detector and the STAS located remotely in the barrack. Both (a) the typical circuit for signals passing from the STAS to the detector and (b) the circuit for signals passing back to the STAS are shown. Note that the VCC bias voltage for the 74128 drivers has to be brought over the data cable to maintain the isolation for the returning signals. 


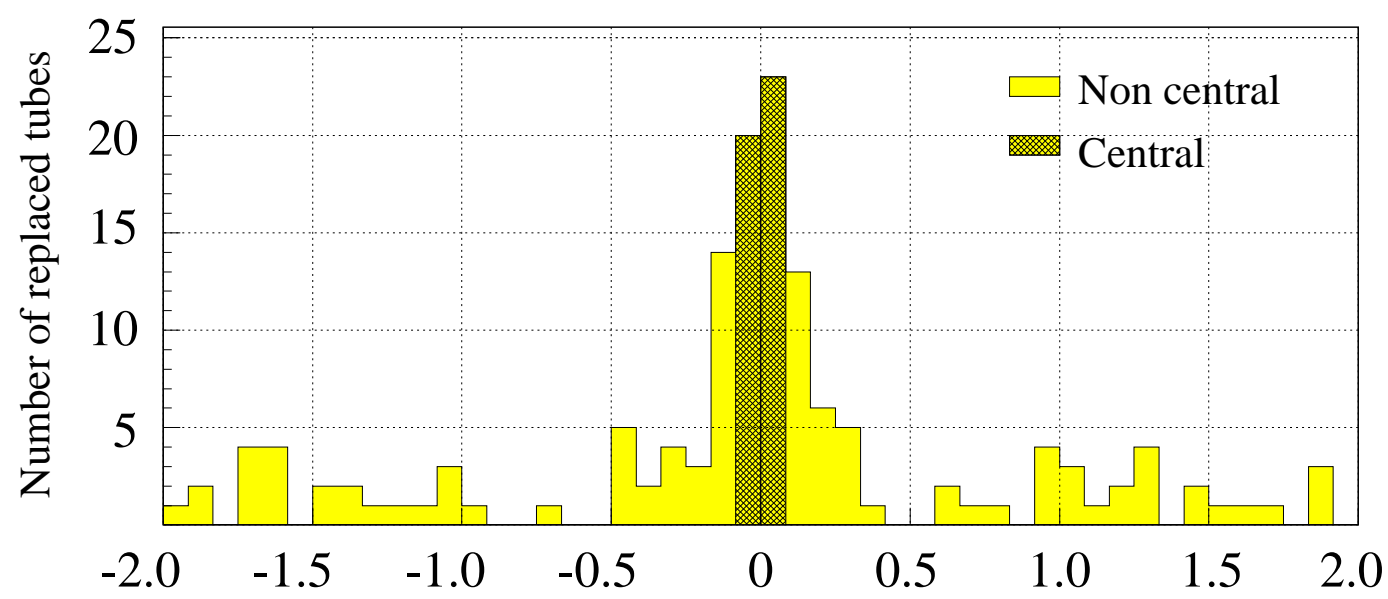

(a) Distance from the beam $[\mathrm{m}]$



Figure 12: (a) The total number of tubes replaced vs. distance from the beam is shown. The positive direction is generally to the side of the greatest flux, the asymmetry being due to the upstream spectrometer magnet. However, this plot also includes the chambers that were deployed horizontally and the positive distance is vertically upward in the lab. These results integrate the experience of 16 planes deployed for five years of data taking. (b) The number of individual chambers replaced, binned by year is shown. The large number of replacements in the first year was influenced by our inexperience with the detector. The relative increase in the last year of running also reflects a conservative decision to make the detector as efficient as possible. 

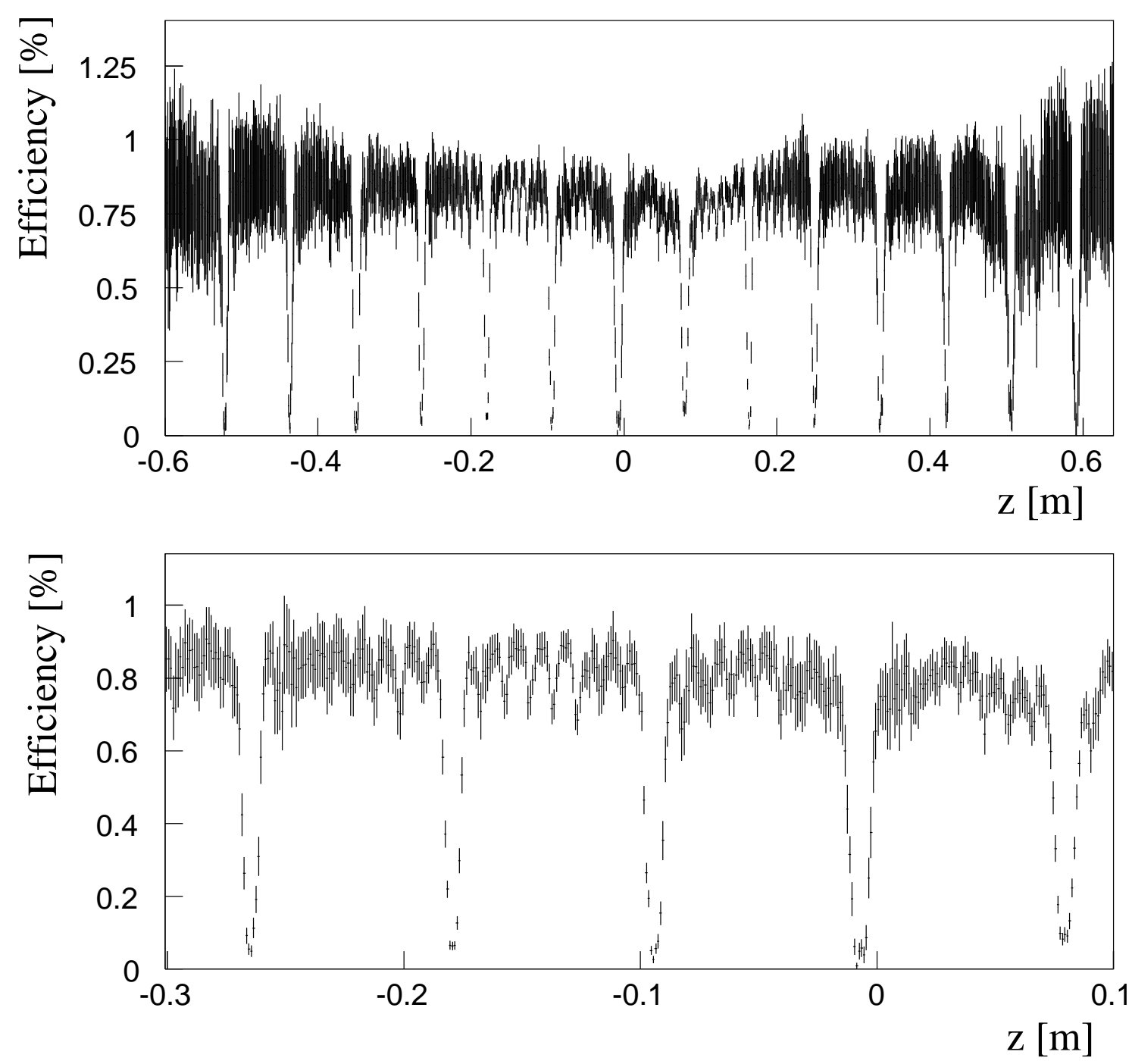

Combined Geometric and Streamer Efficiency

Figure 13: The measured combined geometric and streamer efficiency is plotted for a typical plane of Streamer Chambers as a function of the track's crossing point in the plane of the wires. The view is transverse to the wire direction. The crossing points of the incident muon tracks were determined for this plot from selected events using the other 31 coordinates. The lower plot is an expanded view of the central region of the upper plot. The individual points are plotted for $1 \mathrm{~mm}$ bins and the statistical errors are shown. The internal PVC wall structures are clearly visible. 


\section{References}

[1] "Operation of Limited Streamer Mode Production Line Chambers With Nonflammable Gases," D. Hungerford, et al., NIM A286, 155 (1990). K. Lau, et al., Streamer chamber for muon tracking at the SSC," Proceedings of Symp. on Detector Development for the Superconducting Supercollider, Fort Worth, TX 1990, eds.. T. Dombeck, et al..

[2] See E. Iarocci, NIM 217, 30 (1983) for a review article on the subject.

[3] DAG is the registered trademark for colloidal graphite in methyl isobutyl keytone (MIBK) manufactured by Acheson Colloiden B.V., Scheemda, Holland, The Netherlands.

[4] Private Communication from Roy Weinstein. These developments are considered proprietary and cannot be disclosed in any further detail here.

[5] Tommy Lyons, MIT/SLD Streamer Tube Factory, private communication.

[6] Sintrex is manufactured by Airex S.A., CH 5643 Sins, Switzerland.

[7] SGS Thomson Microelectronics, Subsystems, Engineering and R.F. Division, Centro Direzionale Colleoni, Palazzo Andromeda 3, 20041 Agrate Brianza (Milano), Italy.

[8] Costruzioni Apparecchiature Elettroniche Nucleari S.p.A., Via Vetraia 11, 55049 Viareggio (Italy).

[9] "D779, CMOS Quad Sense Amplifier and Shift Register for Streamer Tube Detector", Data Sheet (No Date), SGS Thomson Microelectronics, Subsystems, Engineering and R.F. Division, Centro Direzionale Colleoni, Palazzo Andromeda 3, 20041 Agrate Brianza (Milano), Italy.

[10] The streamer tube arrays were actually installed in 1991 and preliminary beam trials also occurred during that year. The readout electronics were not available however until 1992, and the evaluations conducted in 1991 were made with readout electronics borrowed from the Gran Sasso LVD experiment as well as from our colleagues from the University of Paduova, whose assistance we gratefully acknowledge.

[11] To avoid the possibility of counting excess current from potential persistent gas discharges, only the HV current values from nominally performing tubes with nominal dark currents are used in making this estimate.

[12] A. Gomez Tato, Ph.D. Thesis, University of Santiago de Compostella, Santiago de Compostella, Spain, 1994. J.J. Saborido, Ph.D. Thesis, University of Santiago de Compostella, Santiago de Compostella, Spain, 1995.

[13] The arrays were essentially $4 \mathrm{~m}$ wide and there were 48 chambers, each with eight $0.9 \mathrm{~cm}$ wide active tube widths. 